REVESCO. Revista de Estudios Cooperativos

ISSN: $1885-8031$

\title{
Motivaciones y efectos sociales de la Economía Colaborativa: el sector del transporte en España como estudio de caso
}

\author{
Carlos Gil de Gómez Pérez-Aradros ${ }^{1}$ y María del Mar Imaz Montes ${ }^{2}$
}

Recibido: 8 de septiembre de 2021 / Aceptado: 27 de diciembre de 2021 / Publicado: 21 de enero de 2022

Resumen. Durante los últimos años, los modelos de negocio surgidos al amparo del paradigma que abre la Economía Colaborativa han experimentado un notable crecimiento, al tiempo que el propio fenómeno de la Economía Colaborativa ampliaba la heterogeneidad de su contenido y, en gran medida, se profesionalizaba. Los efectos de este acontecimiento se perciben en muchos ámbitos, de forma especialmente notoria en el social, donde se clasifican en efectos positivos por un lado y efectos negativos por otro para, utilizando la Teoría del Triple Balance, ponderar la sostenibilidad de estas dinámicas. Paralelamente surge la cuestión: ¿Por qué se participa en los sectores colaborativos? De forma general, un análisis de la literatura académica existente nos conducirá a entender qué motivaciones de tipo social pueden subyacer a la decisión de convertirse en usuario colaborativo; de forma particular, se analiza el sector del transporte colaborativo en España a través de la encuesta "Panel de Hogares" realizada por una institución solvente como es la Comisión Nacional de los Mercados y la Competencia para comprobar qué mueve a estos consumidores, distinguiendo entre los usuarios de un modelo B2C y otro $\mathrm{C} 2 \mathrm{C}$, y proveedores de servicios a serlo, y esbozamos a continuación un breve perfil del consumidor para mejor comprensión de los matices de esta realidad. El resultado de este análisis proporcionará un marco de trabajo a investigaciones teóricas futuras, en tanto construye matrices sociales que contraponen los aspectos positivos frente a los efectos negativos de este paradigma; al tiempo que podría consistir una herramienta práctica en tanto perfila al usuario de transporte colaborativo en España, así como qué le mueve a participar, lo que facilitaría el lanzamiento de campañas destinadas a los grupos sociodemográficos de interés en cuestión.

Palabras clave: Compartir; Participar; Impacto social; Sostenibilidad; Teoría del Triple Balance.

Claves encolit: A14; B55; D16; J46.

\section{[en] Social motivations and social effects of Collaborative Economy: transport sector in Spain as case study}

Abstract. During the last years, bussines models that have risen up under the shelter of paradigm opened by Collaborative Economy have experimented a notable expansion while Collaborative Economy itself was expanding its content's heterogeneity and was greatly turning professional. These occurrence's effects are perceived in different areas, in the social one specially, where we classify positive effects and negative effects for analysing these dynamics'sustainability trought Triple Bottom Line Theory. In the meantime, we set out the question: why do people participate in collaborative sectors? Generally, a current academic literature's analysis will drive us to understand what social motivations can be hidden in the decision of become a collaborative user; particularly, we analyse the collaborative transport sector in Spain using the "Panel de Hogares" survey, made by a solvent institution as the spanish National Commission of Markets and Competition is, for finding what moves these consumers, standing out between users of a $\mathrm{B} 2 \mathrm{C}$ model and users of a $\mathrm{C} 2 \mathrm{C}$ model, and services providers to become so, and following, we make a brief consumer's profile in the interest of a better understanding about every aspects of this reality. The results of this analysis will provide a framework for future theoretical investigations, as long as it builds a social matrix which contrasts positive aspects and negative effects of this paradigm; at same time, it could be a practical tool as long as it defines the user of collaborative transport in Spain as well as what move them to participate, that could make easier to launch compaigns aiming at sociodemographic groups of interest.

Keywords: Share; Participate; Social impact; Sustainability; Triple Bottom Line Theory.

Sumario. 1. Introducción. 2. Marco Teórico. 3. Metodología. 4. Fuentes y datos utilizados. 5. Transporte colaborativo en España. 6. Conclusiones. 7. Referencias bibliográficas.

1 Universidad de Oviedo, España

Dirección de correo electrónico: carlos.gildegomezperez-aradros@asturias.org.

2 Universidad Carlos III de Madrid, España.

Dirección de correo electrónico: delmar.imaz@ gmail.com 
Cómo citar. Gil de Gómez Pérez-Aradros, C.; Imaz Montes, M.M. (2022). Motivaciones y efectos sociales de la Economía Colaborativa: el sector del transporte en España como estudio de caso REVESCO. Revista de Estudios Cooperativos, vol. 140, e79938. https://dx.doi.org/10.5209/reve.79938.

\section{Introducción}

Durante los últimos años, desde la Academia se está prestando una creciente atención al paradigma emergente de consumo conocido como Economía Colaborativa (EC), cuyo principal rasgo característico es el marcado énfasis que pone en la utilidad de uso de los productos y el concepto de compartir, desafiando al modelo convencional de negocio (Botsman y Rogers, 2010; Lamberton y Rose, 2012). Surge del paradigma económico orientado al consumo y, desde el mismo, busca establecer un sistema económico sinérgico orientado al valor (Gold, 2010), la propiedad de los bienes y servicios que se consumen deja de tener tanta importancia en beneficio del propio acceso a los mismos. La decisión de practicar un consumo colaborativo implica crear un valor compartido entre los miembros de la comunidad que sustentan la responsabilidad moral y social de los consumidores basada en la confianza mutua, la cooperación y la solidaridad (Kim y Yoon, 2021).

El interés que despierta la EC, por sus múltiples impactos (económicos, sociales, medioambientales, fiscales o laborales) ha alentado a la literatura académica a preguntarse por las motivaciones que mueven a una persona a convertirse en consumidor y/o proveedor de un servicio colaborativo: ¿Por qué participamos? La explotación de todo el potencial de la EC en aras del desarrollo sostenible únicamente es posible si podemos responder a esta pregunta (Hawlitschek et. al 2018; Möhlmann, 2015).

Varios estudios han tratado de dar respuesta a esta cuestión sin encontrar una razón concluyentemente indiscutible. Téngase en cuenta la enorme amplitud de servicios y modalidades que integran la EC, desde simples intercambios de bienes entre particulares hasta el alquiler de servicios de hospedaje, pasando por transporte de pasajeros, ya sea alquilando un asiento libre en el trayecto de un particular (modelo C2C) o contratando los servicios de un profesional a tales efectos (modelo B2C), que hacen imposible una categorización absoluta de las razones que llevan a un individuo a ofrecer determinados servicios, sus excedentes y a otro a desear adquirirlos o hacer uso de ellos, la importancia relativa de los impulsores varía en función al servicio colaborativo del que se trate debido a su valor económico, al grado de interacción social involucrada y a los efectos ambientales derivados de su uso (Böcker y Meelen, 2017; Cherry y Pidgeon, 2018).

La revisión bibliográfica y los hallazgos empíricos demuestran la ausencia de evidencias categóricas que sean concluyentes y claras (Möhlmann, 2015) en lo relativo a las motivaciones y los efectos en el ámbito del transporte colaborativo en España. Por este motivo el objetivo de esta investigación es múltiple: se pretende llevar a cabo una revisión de la literatura académica relativa a las motivaciones sociales que llevan a participar en alguno de los servicios colaborativos existentes, ofreciendo un documento sintético que resuma las mismas y, a continuación, se lleva a cabo una clasificación de estas motivaciones desde el punto de vista del consumidor y desde el del proveedor. Una vez analizada la literatura académica que se ocupa de las motivaciones para participar en servicios colaborativos (sin especificar sectores ni países), nos centramos en los motivos que mueven, tanto a consumidores como a proveedores, a participar en España en el sector del transporte colaborativo, haciendo una distinción en función a su modelo de negocio entre B2C (de empresa a consumidor) y $\mathrm{C} 2 \mathrm{C}$ (de consumidor a consumidor) para exponer las motivaciones de los consumidores.

En lugar de estudiar la EC como un todo, lo que desvirtuaría las distintas motivaciones y efectos de los diferentes servicios colaborativos, nos hemos centrado en el sector del transporte en España. Esta decisión responde a dos motivos adicionales: por tratarse uno de los servicios con mayor valor económico relativo dentro de la EC y de inversión mundial registrando un $62 \%$ en el período de mayor expansión de este paradigma (CNMC, 2016), así como con mayor grado de protagonismo de la esfera social y medioambiental y por disponer de datos solventes para su estudio, principalmente nos hemos centrado en los proporcionados por la Comisión Nacional de los Mercados y la Competencia (en adelante CNMC) a través de su encuesta "Panel de Hogares", cuya elección está sobradamente justificada en la propia encuesta, pues se adapta al objeto de este estudio recogiendo información sobre motivaciones a nivel social, medioambiental y económico, tanto desde el lado de la oferta como de la demanda del servicio colaborativo de transporte.

Para ello nos servimos de la Teoría del Triple Balance (Böcker y Meelen, 2017) centrándonos en los impulsores económicos, ambientales y sociales que llevan a oferentes y demandantes a ofrecer y presta estos servicios colaborativos y en los efectos producidos por los mismos. Motivaciones y efectos son múltiples y de distinto alcance, siendo de gran utilidad teórica y práctica estructurarlos en tres grandes categorías, siguiendo las premisas de la Teoría del Triple Balance (personas-planeta-beneficio), si bien centrándonos en el ámbito social. La aplicación de la Teoría del Triple Balance se justifica y fundamenta en la asunción de la sostenibilidad como un elemento capital en cualquier práctica, tanto pública como privada, con el fin de 
aumentar la eficiencia económica, social y ambiental (personas, planeta y ganancias) de los recursos disponibles.

Las contribuciones a la literatura académica existente son las siguientes: en primer lugar, al centrar el estudio en el transporte colaborativo en España, determinamos las concretas motivaciones y los particulares efectos de este servicio, sin generalizarlos para la EC en su conjunto, lo que desvirtuaría las posibles diferencias en cada uno de los servicios colaborativos. En segundo lugar, aportamos un análisis y un complemento a la literatura académica existente relativa a dichas motivaciones para la participación en los servicios colaborativos, así como en los efectos de la misma índole que ocasiona tal participación, sistematizado a través de la óptica de la ya mencionada Teoría del Triple Balance por su especial vinculación a las prácticas colaborativas, con la presencia del aprovechamiento de los recursos existentes y la sostenibilidad. En tercer lugar, mostramos evidencias empíricas para el conjunto de España en lo relativo al peso en las motivaciones cuando se actúa desde el lado de la demanda o desde el de la oferta. En cuarto lugar, demostramos que las motivaciones sociales y medioambientales en el uso del transporte colaborativo en el modelo C2C son mucho más relevantes que en modelo B2C. Por último, aportamos el perfil del usuario español de transporte colaborativo en cada una de las principales plataformas de transporte que operan en nuestro país.

\section{Marco teórico}

Resulta imprescindible establecer, con carácter previo al estudio, sus conceptos clave como son la Economía Colaborativa, la motivación, la Teoría del Triple Balance y la sostenibilidad.

La Economía Colaborativa es un concepto emergente en el ámbito de la empresa y la economía que plantea la aparición de nuevas oportunidades de negocio y de generación de ideas basadas en las tecnologías de la comunicación, que eliminan barreras facilitando la conexión entre participantes y reducen costes e incertidumbres asociadas a la actividad comercial (Schor y Fitzmaurice, 2015). De forma recurrente, se asocian a este modelo económico aspectos como la confianza, la cooperación, una mayor redistribución de riqueza entre sus participantes, o la búsqueda de justicia en los intercambios realizados, entre otros, que hacen que muchas veces se la vincule con el término "social", incluyendo en el mismo paraguas a las experiencias de Economía Colaborativa y economía social (Díaz- Foncea et al., 2016). No es sencillo conceptualizar de forma global la EC, pues recibe múltiples denominaciones que identifican realidades con rasgos comunes pero que también presentan diferencias (Dip et al., 2020), entre otras, gig economy, platform economy o peer-to-peer economy. Así, a pesar de que diferentes autores han centrado su trabajo en tratar de dotar a la Economía Colaborativa de dicho concepto (Frenken y Schor, 2017), en la actualidad continúa sin existir una terminología unánime para referir este fenómeno. A fin de evitar estos debates terminológicos que, si bien ricos y necesarios, exceden el objeto del presente análisis, hemos optado por una definición lo más aséptica e institucional posible, enmarcarnos en la conceptualización de la Comisión Europea (2016: 3) que refiere la EC como "modelos de negocio en los que se facilitan actividades mediante plataformas colaborativas que crean un mercado abierto para el uso temporal de mercancías o servicios ofrecidos a menudo por particulares", con o sin ánimo de lucro, y donde se pueden distinguir tres tipos de participantes: los que prestan el servicio compartiendo activos, recursos, tiempo y/o competencias, bien sean particulares o profesionales; los usuarios de dichos servicios; y finalmente, intermediarios que conectan a unos y otros, a través de plataformas en línea.

Con motivación referimos una serie de impulsos, deseos o estímulos que fomentan un comportamiento determinado (Soler y Chirolde, 2010), destinado a la satisfacción de ciertas necesidades, lo que implica que una vez satisfecha la necesidad, se reduce el impulso que motivó la conducta (Ferrell y Hirt, 2003; Robbins y Coulter, 2005). Para analizar el comportamiento subyacente al consumo colaborativo, estudios previos han señalado que los participantes están motivados por un conjunto de valores multidimensionales (Martin y Upham, 2016). La mayoría de estos análisis han optado por emplear la teoría de la autodeterminación a fin de categorizar estas motivaciones en intrínsecas (como por ejemplo, los valores de sostenibilidad) y extrínsecas (como por ejemplo, el beneficio económico). Nosotros, con el fin de dotar de un papel protagonista a la idea de sostenibilidad, motivador intrínseco clave según Hamari el al. (2016), hemos aplicado la Teoría del Triple Balance o Triple Cuenta de Resultados (TBL) que permitió la inserción de los principios de sostenibilidad en las organizaciones empresariales (Elkington, 2001), incorporando elementos como la equidad social, la responsabilidad medioambiental y el desarrollo económico (personas, planeta y ganancias) que interactúan y entran en conflicto en torno a la citada sostenibilidad (Hindle, 2009), entendida como la interconexión de los tres ámbitos. Aunque este modelo fuera inicialmente ideado para empresas, actualmente es posible desagregar sus elementos en uno o varios motivadores para consumir o proveer un servicio colaborativo.

El propio concepto de sostenibilidad es amplio y variado en tanto que depende del enfoque que cada autor quiera otorgarle. Sin pretender profundizar en una compleja conceptualización del mismo, por un lado, se 
encuentra la llamada sostenibilidad fuerte que parte de la dimensión ecológica, y se basa en que el capital natural es único y no puede ser reemplazado por el capital humano; por otro, se encuentra la denominada sostenibilidad débil, la cual tiene un enfoque más económico, lo que permite la sustitución mutua de capital entre las tres dimensiones (Luffiego, 2000; Sánchez, 2009; Ríos-Osorio et. al., 2013).

\section{Metodología}

La metodología a aplicar en este estudio consiste en la revisión de literatura académica sobre las motivaciones de tipo social que influyen en la decisión, tanto de consumidores como de proveedores, de participar en negocios colaborativos de forma general, sin distinguir entre plataformas ni sectores; y sobre los efectos, también de tipo social, que está ocasionando el desarrollo de este paradigma, clasificándolos en positivos y en negativos. La revisión bibliográfica se ha desarrollado sistemáticamente haciendo uso de reconocidas bases de datos como Proquest, Scopus o Bussines Source Complete, restringiendo los criterios de búsqueda a revistas científicas y profesionales (fuentes primarias) escritas en español y en inglés, con términos limitados al objeto de estudio (es decir, "motivaciones de la EC", "efectos de la EC", "EC y sociedad", "Impacto social de la EC", "participación en EC", etc). Este procedimiento se llevó a cabo durante agosto de 2021, de forma independiente por los dos investigadores y, recopilados todos los textos, se sintetizaron éstos conjuntamente y se conectaron sus premisas para ofrecer la clasificación pretendida. De esta búsqueda fueron extraídos setenta y dos artículos. De forma adicional, también se recurrió a fuentes secundarias para completar la perspectiva (tales como monografías sobre derecho del trabajo a fin de comprender la notable problemática derivada de las relaciones laborales en el marco colaborativo); así como a documentos institucionales, principalmente de la Unión Europea.

Paralelamente, analizando la encuesta "Panel de Hogares" elaborada por la Comisión Nacional de los Mercados y la Competencia, se desarrolla un estudio del caso concreto del transporte colaborativo en España, al contar con datos relativos a las motivaciones de los participantes en EC para optar por consumir o proveer este tipo de servicios. De este cuestionario, con respuestas múltiples y no excluyentes, se agruparán las contestaciones en función a las tres tipologías de motivación extraídas de la aplicación de la Teoría del Triple Balance: económicas, ambientales y sociales, para las cuestiones relacionadas con el sector objeto del análisis.

La función de la Teoría del Triple Balance o Triple Cuenta de Resultados (TBL), como ya se ha podido esbozar, consiste en contribuir a conformar criterios homogéneos y objetivos de fácil comparación, que puedan ayudar a entender los motivos que impulsan a hacer uso de este modelo, así como los efectos del mismo. Además, en los últimos años esta teoría ha ido captando la atención de un creciente número de estudios, justificado ello en factores como la escasez de recursos productivos y la necesidad de optimizar los procesos no sólo en términos económicos, sino especialmente en sus aspectos ambientales y sociales; los cuales han impulsado en la actualidad el estudio de modelos de sostenibilidad (Pereira y Martins, 2021).

En el presente estudio vamos a analizar sustancialmente el nivel social, para lo cual se plantea la matriz de la Teoría del Triple Balance con aspiración a convertirse en un mecanismo que permita evaluar la motivación social, por ello, su diseño se estructura en torno a la misma e incluye a las partes que pueden participar en servicios colaborativos (demandantes y oferentes). Las interacciones que pueden darse entre los distintos actores y motivaciones son las recogidas en la tabla 1.

Tabla. 1. Matriz de motivaciones sociales para consumidor y proveedor de servicios colaborativos.

\begin{tabular}{|c|c|c|}
\hline Motivaciones & Consumidor & Proveedor \\
\hline $\begin{array}{l}\text { Motivos } \\
\text { sociales }\end{array}$ & $\begin{array}{l}> \\
\text { Conocer a nuevas personas, } \\
\text { nueva experiencia social, } \\
\text { conectar con otros } \\
>\end{array}$ & $\begin{array}{l}\text { Conocer a nuevas personas, } \\
\text { nueva experiencia social, } \\
\text { conectar con otros. }\end{array}$ \\
\hline
\end{tabular}

Fuente: Elaboración propia aplicando la Teoría del Triple Balance a la literatura académica sobre motivaciones para participar en EC. 
Por lo que respecta a los efectos sociales de la EC, se plantea la siguiente matriz (tabla 2) que pretende convertirse en un mecanismo que proporcione soluciones a los servicios colaborativos, con el fin de evaluar la sostenibilidad a nivel social, por ello, su diseño se estructura en torno los mismos e incluye a las partes que se pueden ver afectadas por las actividades de estos servicios colaborativos. En relación a las partes implicadas, hemos incluido a las siguientes: directivos, accionistas/inversores, trabajadores, consumidores/proveedores, humanidad/futuras generaciones y medioambiente. Las interacciones que se han de dar entre los distintos elementos aparecen recogidas en la matriz.

Tabla. 2. Matriz de efectos sociales de la EC.

\begin{tabular}{|c|c|c|c|c|c|c|}
\hline & : & 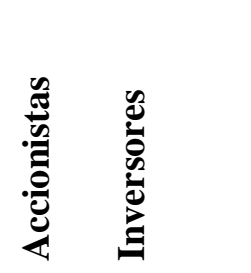 & 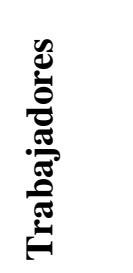 & 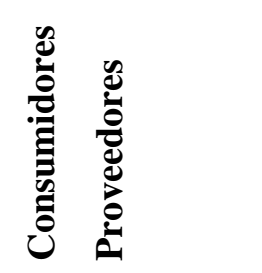 & 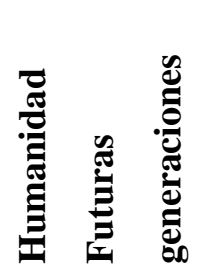 & 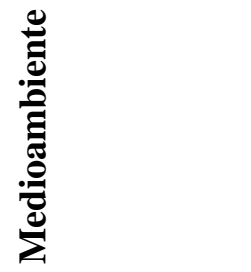 \\
\hline $\begin{array}{l}\text { Efectos } \\
\text { sociales + }\end{array}$ & $\begin{array}{l}\text { Responsabilida } \\
\text { d social }\end{array}$ & Inversión ética & $\begin{array}{l}\text { Desarr } \\
\text { ollo } \\
\text { profesi } \\
\text { onal }\end{array}$ & $\begin{array}{l}\text { Empoderamie } \\
\text { nto de } \\
\text { consumidores/ } \\
\text { proveedores }\end{array}$ & $\begin{array}{l}\text { Desarrollo } \\
\text { socialmente } \\
\text { sostenible }\end{array}$ & $\begin{array}{l}\text { Respeto social } \\
\text { y } \\
\text { medioambient } \\
\text { al }\end{array}$ \\
\hline $\begin{array}{l}\text { Efectos } \\
\text { sociales - }\end{array}$ & $\begin{array}{l}\text { Prácticas } \\
\text { sociales } \\
\text { reprochables }\end{array}$ & $\begin{array}{l}\text { Pérdida } \\
\text { inversión } \\
\text { social }\end{array}$ & $\begin{array}{l}\text { Desigu } \\
\text { aldad }\end{array}$ & $\begin{array}{l}\text { Asimetría de } \\
\text { poder }\end{array}$ & $\begin{array}{l}\text { Descohesió } \\
\text { n social }\end{array}$ & $\begin{array}{l}\text { Desconexión } \\
\text { sociedad-ma }\end{array}$ \\
\hline
\end{tabular}

Fuente: Elaboración propia a partir de la revisión de literatura académica sobre efectos de la EC

En relación a la posibilidad que nos proporciona la herramienta TBL a través de su matriz, una vez cuantificados los efectos sociales positivos y negativos, podremos valorar la sostenibilidad social de los servicios colaborativos, otorgándole tal consideración si los primeros son mayores que los segundos o insostenible si los efectos adversos son mayores, más numerosos o más intensos. Por ello, es necesario concretar los beneficios de los servicios colaborativos (efectos positivos) dentro de la matriz de la forma que se ilustra en la tabla 3.

\section{Fuentes y datos utilizados para el estudio}

Atendiendo al objeto múltiple de este estudio, las fuentes utilizadas también lo son. Así, como se ha adelantado, el análisis de la encuesta "Panel de Hogares" publicada por la CNMC en aras de una caracterización del proveedor y consumidor español de transporte colaborativo (sin perjuicio de recurrir a otras encuestas como Statista Global Consumer Survey para completar la visión del consumidor), irá precedido de una revisión y clasificación de la literatura académica sobre motivaciones y sobre efectos, tanto desde el lado de la oferta como del de la demanda, de tipo social

\subsection{Literatura académica: motivaciones sociales}

Ante todo, destacar la dificultad intrínseca a los estudios sobre factores motivacionales. Esto es debido a la inclinación hacia la especialización de la mayoría de los estudios existentes, centrados en servicios o plataformas concretas, que hace compleja la comparación de resultados y el trazado de tendencias (Birke, 2009; Nevo et al., 2016; Peitz y Waldfogel., 2012). En este sentido, destacar las conclusiones de Böcker y Meelen (2017), que tras realizar una comparativa entre motivaciones para participar en diversas plataformas colaborativas de distintos sectores, coligen que las motivaciones son diferentes en cada caso y que no podría concebirse la EC como un fenómeno homogéneo integrado por un único tipo de mercado. No obstante, es posible destacar algunos rasgos propios de diferentes actividades: en el caso de aquellos servicios que cuentan con un alto componente de interacción personal, la motivación social sería predominante (Patiño et al., 2017); cuando se comparten bienes de alto valor, las motivaciones económicas serían fundamentales; y, concretamente para el sector del transporte colaborativo, las razones medioambientales serían especialmente relevantes (Böcker y Meelen, 2017); sin que el papel protagonista de una tipología motivacional sea óbice para la presencia de las otras dos (Scaraboto, 2015). 
Dentro de las motivaciones sociales que impulsan a participar como consumidor de servicios colaborativos, destaca principalmente la consecución de nuevas experiencias sociales, conocer a nuevas personas, conectarse con otros y el sentimiento de parte de una comunidad derivado de sus interacciones (Barnes y Mattsson, 2017; Tussyadiah, 2016; Tussyadiah y Pesonen, 2018), así como el capital social, cuya dimensión de poseer un objetivo compartido sería el motivador más fuerte (Kim y Yoon, 2021). Otros estudios inciden más aún en la idea de pertenencia a la comunidad como motivador (Närvänen et al. 2013; Peterson, 2008; Albinsson y Perera, 2012), junto con la coproducción colectiva (Peters et al., 2012) y el desarrollo personal (Abhari et al, 2019).

Se han señalado valores de tipo altruista, como ayudar a otros (Kankanhalli et. al., 2005), en la decisión de participar en EC, así como otros de corte más individualista, tales como la autenticidad, la novedad, la personalidad y el carácter distintivo que se identifican con estos usos y consumos (Guttentag et al., 2018).

Respecto a la decisión de proveer servicios colaborativos, las motivaciones sociales también se encuentran muy presentes. En este sentido, se ha detectado la necesidad de socializar y establecer vínculos emocionales con otros (Fischer et al., 2019) como fuerte motivador.

\subsection{Literatura académica: efectos sociales}

A pesar de la preeminencia otorgada a los efectos económicos de la EC en la literatura académica, ésta "no se ciñe exclusivamente al ámbito económico, sino que hace hincapié en aspectos sociales" (Parlamento Europeo, 2017 a 9), dado que la actividad colaborativa tiene una amplia vertiente que se desarrolla en entornos de proximidad, a menudo sin ánimo de lucro, y tiene una dimensión local, pues aunque inseparable de la dinámica de Internet, utiliza la web como un mecanismo de colaboración pero su objetivo es generar acción en el mundo real (Botsman y Rogers, 2010).

En la dimensión social, se distinguen tanto efectos positivos como negativos de este modelo.

De forma positiva, puede destacarse que la EC se habría convertido en un mecanismo para satisfacer nuevas necesidades sociales (Cherry y Pidgeon, 2018; Sutherland y Jarrahi, 2018; Toni et al., 2018).

Binninger et. al. (2015) destacan en cuatro ideas el impacto positivo del paradigma colaborativo en la sociedad: el principio de masa social crítica, condición necesaria para lograr la sostenibilidad social y medioambiental del modelo, presupone que el número de participantes en los intercambios es lo suficientemente grande como para satisfacer las necesidades del consumidor en términos de elección; la "compartición" entre iguales que no se conocen del exceso de capacidad inactiva en los bienes; la creencia en la posibilidad de gestión comunitaria de los recursos (Ostrom, 2010); y la confianza mutua, factor clave del éxito de los sistemas de intercambio. De esta dinámica de intercambio entre desconocidos puede derivar un incremento de la cohesión social (Botsman y Rogers, 2010; Cherry y Pidgeon, 2018; Ferrari, 2016) y en la acumulación de capital social, que a su vez incide en la motivación para participar especialmente estimulando el propio disfrute de la experiencia colaborativa (Kim y Yoon, 2021). Ello está íntimamente relacionado con la producción de una sensación de comunidad que busca una mejor forma de gestionar los bienes en atención al beneficio colectivo: se conoce a gente nueva, se obtienen experiencias sociales nuevas, se conecta con otros y se forma parte de dicha comunidad (Barnes y Mattson, 2017; Tussyadiah, 2016; Tussyadiah y Pesonen, 2018), motivador y efecto al mismo tiempo. En esta misma línea, algunos autores van más allá y hablan de "comunidades tribales de consumo colaborativo" (Barnes y Mattsson, 2016: 6), consolidadas por medio de un nuevo lugar, procedimiento y herramientas de consumo que aumenta rápidamente el número de miembros de la tribu. Éstos, por medio de la interacción, crean significados, comparten valores y son incluso capaces de boicotear o crear campañas de impulso de bienes, usos o prácticas comunitarias (Canniford, 2011; Cova y Cova, 2002; Cova y White, 2010; Muniz y O'Guinn, 2001). Para entender estas tribus, destacar cuatro características que sintetizan su dinámica (Canniford, 2011): multiplicidad, que no excluye otras prácticas o adhesiones; integración, por la se otorga valor al vínculo social; fugacidad, donde las tribus cambian a medida que lo hace el entorno; e innovación, generadora de nuevas formas de emprendimiento social y económico.

En definitiva, se crean nuevos lazos sociales (Bicchieri et. al. 2004; Benkler, 2004; Gualtieri, 2014; Aparici y García, 2018), vinculado a los valores propios de la EC, más democráticos y respetuosos con el interés general (Scholz, 2014), y ayuda a las personas más necesitadas atendiendo a los colectivos cercanos al tiempo que se convierte en una alternativa a modelos impersonales de consumo masivo (Germann, 2013). En este sentido, Rifkin (2014) pronostica para la segunda mitad del siglo XXI un nuevo paradigma colaborativo, basado en una conexión mundial y la gestión colectiva, que desplazará al capitalismo tal y como lo entendemos.

Paralelamente, se destaca un empoderamiento de los consumidores (Cherry y Pidgeon, 2018; Martin, 2016), un poder adquirido frente al proveedor tradicional, compitiendo con ellos (Owyang et al., 2013), y la propia capacidad de transformarse en proveedor, en consumidor o en ambas figuras al mismo tiempo (Ferrer y Paniagua, 2017). 
Por otro lado, algunos investigadores detectan la existencia de efectos negativos de la EC en el ámbito social, de manera protagonista ligados al concepto de precariedad ${ }^{3}$, ya que al amparo del paradigma colaborativo han proliferado plataformas que, sirviéndose de las zonas grises del derecho, fomentan fórmulas de negocio basadas en el trabajo precario (Goldin, 2019) y un empeoramiento de la calidad del empleo respecto a las condiciones en que prestan sus servicios los trabajadores del modelo clásico (Rogers, 2017). Esta gig economy trasciende el espíritu inicial de intercambio y colaboración entre iguales que fomenta la EC, y tiende a desdibujar las líneas que separan lo profesional de lo personal (Sundararajan, 2016) induciendo, además, a los operadores del servicio a percibirse en la práctica como una microempresa con capacidad propia de acción y de decisión, lo cual no puede sostenerse respecto a todos los prestadores de servicio en este modelo. Estas plataformas colaborativas amplificarían los peores excesos del modelo económico dominante, desarrollando así una versión exagerada de los ideales del neoliberalismo (Morozov, 2013) que, buscando la desregulación del mercado, crearían espacios formales de competición habitados por "empresarios de sí mismos" (Foucault, 2007: 158), por "prosumidores" dispuestos a valorizar como sea sus activos personales en el mercado digital (Gil, 2016).

En relación a Uber (Kilhoffer et al., 2017) la independencia de los conductores es inexistente ya que no pueden negociar con los pasajeros y aceptan viajes según los términos impuestos por la empresa. Una réplica de esquemas tradicionales propios de la relación laboral pero, al tiempo, sustrayendo de la protección social a la persona que ejecuta el servicio para una plataforma (Ballester, 2019).

El Parlamento Europeo (2017 : 10), ante el aumento de las tasas de trabajo autónomo y el porcentaje de trabajadores con un segundo empleo, alertaba a los Estados miembros sobre la necesidad de abordar de "forma más apropiada la dimensión social de la economía de las plataformas ofreciendo un análisis exhaustivo y los datos correspondientes sobre las nuevas formas de empleo, supervisando el entorno regulador en evolución y fomentando el intercambio de buenas prácticas entre los Estados miembros, con el fin de ofrecer una respuesta a los problemas sociales relacionados con esta nueva economía". Esta advertencia ya había sido realizada previamente por la misma institución, apuntando la importancia de "garantizar medios suficientes para ofrecer a escala nacional una protección social adecuada a todas las personas, independientemente de la forma de empleo en la que ejerzan su actividad y tanto si se trata de relaciones laborales convencionales como no convencionales o de trabajo por cuenta propia" (Parlamento Europeo, 2017b: 19).

Además, en sentido contrario a lo expresado por otros autores, algunos estudios concluyen que la confianza no está generando un mayor bienestar de los consumidores en este paradigma (Munar y Gyimóthy, 2013) ni siquiera el mencionado empoderamiento de los mismos, ya que la percepción de participación y soberanía del consumidor no sería real: las valoraciones y comentarios de otros consumidores, en los cuales se basan los sistemas de confianza que rigen las dinámicas de la EC, no estarían completamente respaldados por la transparencia y fiabilidad deseables (Wosskow, 2014), sino que se ven influidos por actitudes de "vigilancia y castigo" entre usuarios/as (Pick, 2012); y, además, la tecnología que facilitaría este empoderamiento, en realidad, no tendría el pretendido matiz emancipador, reproduciría nuevos tipos de asimetría de poder y de jerarquía social (Labrecque et al., 2013), pues los intermediarios que propician la conexión entre los usuarios colaborativos que quieren realizar el intercambio (Zelizer, 2010) serían los sujetos que verdaderamente marcan las reglas, garantías y responsabilidades de la transacción, cuestionándose así la equidad que se le presupone a los intercambios propios de la EC, pues estos intermediarios ejercerían realmente el poder y el control del negocio (Dredge y Gyimóthy, 2015), replicando consecuentemente los patrones de privilegios preexistentes (Cagle, 2014) pues en muchos casos se trata de actores económicos profesionales que desvirtúan el espíritu colaborativo de la plataforma (Gil y Sequera, 2018). Además, la satisfacción de deseos o necesidades individuales se desconecta de las instituciones locales para orientarse hacia plataformas globales: el viaje en tren o autobús es sustituido por el uso de Blablacar, por ejemplo, movimiento que puede ser percibido como liberador o empoderador para el individuo, pero a medio plazo tiene un efecto desestructurante sobre la sociedad, destruyendo la sostenibilidad de las instituciones de la economía local (González de Rivera, 2019), como dándose una pérdida de líneas o frecuencias de transporte público debido a la menor ocupación de las mismas, por seguir con el ejemplo planteado.

Así mismo, el aspecto positivo de la cohesión social también ha sido discutido, autores consideran que ésta podría verse amenazada por las externalidades negativas que pueden surgir para terceros (Böcker y Meelen, 2017; Frenken y Schor, 2017), por ejemplo, molestias para los vecinos de alojamientos colaborativos $^{4}$ y, en la misma línea, una turistificación de los barrios del centro histórico de muchas ciudades

Entiéndase por tal, la vulnerabilidad e inseguridad laboral que, yendo más allá del tipo de contrato, condiciona la posibilidad real de emancipación y desarrollo personal de los trabajadores (Lacalle, 2009). Son relaciones de empleo en las que los trabajadores quedan excluidos de la protección social propia de un Estado de Bienestar y de la capacidad de hacer valer sus derechos, y de las que deriva un salario insuficiente para llevar una vida decente y unas condiciones que imposibilitan hacer planes de futuro (McKay et al., 2012: 84).

4 Citado el alojamiento por ser especialmente sensible a estas situaciones. A título ilustrativo, véase "Así fastidia la vida a unos vecinos del centro de Madrid un piso turístico ilegal ante la pasividad administrativa", noticia del 29.07.2021 de ElDiario.es: 
europeas (Gottlieb, 2013). Dicha turistificación, como efecto social negativo constatado respecto a la expansión del sector de alojamiento colaborativo, implica a su vez otros efectos sociales nocivos, tales como el desplazamiento espacial de los estratos sociales menos favorecidos debido a una revalorización del mercado inmobiliario local (Pixová y Sládek, 2016), la desposesión simbólica y material de los vecinos (Janoschka y Sequera, 2016), una creciente marginalización de presencias, prácticas y consumos 'no deseados' mediante políticas gubernamentales de tolerancia-cero (Sequera, 2017), tensiones en la convivencia derivadas de un modelo de ocio hedonista y mercantilizado durante las horas nocturnas (Nofre et al, 2018), la desaparición de los comercios de barrio dedicados a la venta al por menor y la manufactura, que se ven reemplazados por otros dirigidos al consumo del turista (González y Waley, 2013); y una íntima relación entre este fenómeno, la crisis de la vivienda (Mertens, 2017) y otros procesos urbanos como la gentrificación o la implementación de 'políticas de gentrificación' (Sequera, 2017; Wachsmuth y Weisler , 2017).

Por último, se han constatado patrones de discriminación social como efecto de la EC, debido al propio perfil del usuario colaborativo, esto es, individuo con alto capital económico, cultural y digital, geográficamente metropolitano o residente en centros urbanos, frente a otros estratos sociales inferiores o residentes periféricos que no tienen la posibilidad de acceso a este mercado (Menor-Campos et al, 2019; Frenken y Schor, 2017; Kassan y Orsi, 2012) ya sea por la falta de medios técnicos, por la insuficiencia de conocimientos para su uso, carecen de capital inactivo para intercambiar o por la ausencia de mercado en su entorno, lo que contribuye a aumentar la desigualdad social y económica (Cherry y Pidgeon, 2018; Frenken, 2017; Schor, 2017) y un incremento de la discriminación (Frenken y Schor, 2017), a la vez que mantiene desigualdades preexistentes como las basadas en la raza (Cansoy y Schor, 2016; Schor, 2017; Schor y Attwood-Charles, 2017; Smith, 2016). Incluso algunos estudios empíricos señalan que dentro del paradigma colaborativo tienen lugar discriminaciones por condición de raza (Edelman et al., 2017) y de orientación sexual (Ahuja y Lions, 2017), que otras investigaciones desmienten o cuestionan (Hajibaba y Dolnicar, 2017; Cui et al., 2016).

La tabla 4 ilustra este debate sobre la pluralidad de efectos sociales que implica el paradigma colaborativo, clasificándolos en positivos y en negativos, como resultado de nuestra revisión de literatura académica.

Tabla. 3. Efectos sociales de la Economía Colaborativa.

\begin{tabular}{|c|c|}
\hline Efec & \\
\hline $\begin{array}{l}\text { Crea nuevos lazos sociales (Bicchieri et. al. 2004; } \\
\text { Benkler, 2004; Gualtieri, 2014; Aparici y García, } \\
\text { 2018) }\end{array}$ & $\begin{array}{l}\text { Ausencia de una adecuada protección social } \\
\left(\text { Parlamento Europeo, } 2017^{\mathrm{a}}\right) \\
\begin{array}{l}\text { Las plataformas generan trabajo precario y } \\
\text { dependiente (Ballester, 2019) }\end{array}\end{array}$ \\
\hline ogers, & Turistificación (Gottlieb, 2013) \\
\hline $\begin{array}{l}\text { ta la solidaridad, la colaboración y la idea } \\
\text { unidad, (Cherry y Pidgeon, 2018; } \\
\text { d y Jarrahi, 2018; Toni et al., 2018) }\end{array}$ & empoderamiento social (Munar \\
\hline $\begin{array}{l}\text { Aumenta la acción colaborativa (Bostman y } \\
\text { Rogers, 2011) }\end{array}$ & cial \\
\hline $\begin{array}{l}\text { Fomenta la masa social crítica, la "compartición" } \\
\text { entre iguales, la gestión comunitaria de los } \\
\text { recursos y la confianza mutua (Binninger et. al., } \\
\text { 2015) }\end{array}$ & $\begin{array}{l}\text { vas definen las reglas, las } \\
\text { ades y los riesgos de las } \\
\text { 0) }\end{array}$ \\
\hline $\begin{array}{l}\text { Crea "comunidades tribales de consumo } \\
\text { colaborativo" (Barnes y Mattsson, 2016). } \\
\text { No se produce discriminación (Hajibaba y } \\
\text { Dolnicar, 2017; Cui et al., 2016). }\end{array}$ & $\begin{array}{l}\text { Genera discriminación social (Frenken y Schor, } \\
\text { 2017), así como por raza (Edelman et al., 2017) y } \\
\text { por orientación sexual (Ahuja y Lions, 2017). }\end{array}$ \\
\hline $\begin{array}{l}\text { Proporciona una visión moral y socialmente } \\
\text { responsable (Kim y Yoon, 2021). }\end{array}$ & $\begin{array}{l}\text { ca (Cherry } \\
17)\end{array}$ \\
\hline Crea lazos comunitarios y ayuc & IV as (DUCh \\
\hline
\end{tabular}

https://www.eldiario.es/madrid/somos/noticias/fastidia-vida-vecinos-centro-madrid-piso-turistico-ilegal-pasividad-

administrativa 1 8173966.html (acceso 27.08.2021). Derivado de esta problemática, plataformas colaborativas como Airbnb han habilitado espacios de asistencia a los vecinos en caso de problemáticas derivadas de la convivencia con los inquilinos del servicio: https://www.airbnb.es/neighbors. 


\begin{tabular}{|l|l|}
\hline más necesitadas y cercanas (Germann, 2013) & $\begin{array}{l}\text { 2017; Frenken y Schor, 2017). } \\
\text { Destruye la sostenibilidad de las instituciones de la } \\
\text { economía local (González de Rivera, 2019) }\end{array}$ \\
\hline $\begin{array}{l}\text { Proporciona transparencia y cercanía (Aparici y } \\
\text { García, 2018) }\end{array}$ & $\begin{array}{l}\text { Perpetúa privilegios y niegan bienestar para otros } \\
\text { (Cagle, 2014). }\end{array}$ \\
\hline $\begin{array}{l}\text { Empodera a los consumidores (Cherry y Pidgeon, } \\
\text { 2018; Martin, 2016) }\end{array}$ & $\begin{array}{l}\text { Excluye a colectivos sin capital inactivo que } \\
\text { intercambiar (Kassan y Orsi, 2012) }\end{array}$ \\
\hline
\end{tabular}

Fuente: elaboración propia.

\subsection{Datos. Panel de Hogares de la CNMC}

Para el estudio de caso, hemos tomado los datos de la encuesta "Panel de Hogares" elaborada por la CNMC. Si bien esta información data de 2019, es la más reciente sobre EC de la que dispone este organismo y, además, es el período más idóneo para el estudio de este fenómeno ya que la información relativa a los años 2020 y 2021 estaría altamente influida por la pandemia COVID-19, las restricciones impuestas y la propia dinámica del distanciamiento social, de tal forma que no permitiría un análisis de este paradigma en la esfera social sin monopolizarlo ${ }^{5}$. Este instrumento consiste en una investigación longitudinal sobre una muestra de individuos de diez años o más residentes en viviendas familiares en el territorio español (excepto Ceuta y Melilla) con la finalidad de recabar información sobre la disponibilidad de los servicios y equipos, los consumos y gastos, y los hábitos y percepciones de los usuarios del segmento residencial respecto a diferentes sectores supervisados por la CNMC, tales como energía o telecomunicaciones, con una periodicidad semestral, a través de cuestionarios postales $(66,7 \%$ de los hogares) o web $(33,3 \%$ de los hogares) de respuesta múltiple sobre su disponibilidad, uso y percepciones de los servicios. En el segundo semestre de 2019, encuesta que hemos utilizado, la muestra neta de los cuestionarios fue de 4.861 hogares y de 9.058 individuos dentro de los parámetros geográficos y personales referidos (CNMC, 2019).

En cuanto a motivación para participar en la EC, centrándonos en las sociales, las preguntas concretas a destacar serían las siguientes:

1.- ¿Qué le ha llevado a utilizar estos servicios?

- Desplazarme en coche con conductor por la ciudad (ej.: Cabify, Uber)

- No uso

- Tienen un precio más ventajoso

- Ofrecen mayor calidad de servicio que otros servicios tradicionales

- Son servicios que se ajustan mejor a mis necesidades y horarios

- Ofrecen un servicio que no encuentro en el mercado

- Me evitan tener que comprar algo que no voy a utilizar mucho

- Son más sostenibles medioambientalmente

- Las plataformas me permiten elegir con más información entre los ofertantes y eso me genera confianza

- Es un medio para conocer personas e intercambiar experiencias

- Los servicios se contratan de manera más cómoda y ágil que los servicios tradicionales

- Otros

- Desplazarme en coche a otras ciudades aprovechando el viaje de un conductor particular (ej.: Blablacar, Amovens, Carpooling)

- No uso

- Tienen un precio más ventajoso

- Ofrecen mayor calidad de servicio que otros servicios tradicionales

- Son servicios que se ajustan mejor a mis necesidades y horarios

- Ofrecen un servicio que no encuentro en el mercado

- Me evitan tener que comprar algo que no voy a utilizar mucho

Según datos de EY-Parthenon (2020), el 49\% de los encuestados afirmaban que no retomarían el uso de vehículos compartidos hasta que España se recobrase de la crisis sanitaria, un $28 \%$ afirmaba esperar a la generalización de la vacuna y el $23 \%$ sostenía que lo haría tan pronto como las restricciones se lo permitiesen. 
- Son más sostenibles medioambientalmente

- Las plataformas me permiten elegir con más información entre los ofertantes y eso me genera confianza

- Es un medio para conocer personas e intercambiar experiencias

- Los servicios se contratan de manera más cómoda y ágil que los servicios tradicionales

- Otros

2.- ¿Qué le ha llevado a ofrecer estos servicios?

- Compartir plazas libres de mi coche (ejemplos: Blablacar, Amovens, Carpooling)

- No oferto este servicio

- Me permite aumentar mis ingresos aprovechando recursos que tengo disponibles

- Las plataformas me permiten ofrecer estos servicios de manera cómoda y ágil

- Las plataformas contienen información de los demandantes y eso me genera confianza

- Es una contribución a la sostenibilidad medioambiental

- Es un medio para conocer personas e intercambiar experiencias

- Otros

\section{Transporte colaborativo en España}

Aunque no debe olvidarse que las razones ambientales pueden ser especialmente importantes en relación con el uso compartido de automóviles (Böcker y Meelen, 2017; Prettenthaler y Steininger, 1999), es especialmente relevante que ello implica cierta interacción social antes del viaje pero, principalmente, durante el mismo.

Según los datos proporcionado por el "Panel de Hogares", exponemos a continuación la situación del sector del transporte en España tanto desde el lado de la demanda como del de la oferta. Puntualizar que, respecto a los consumidores, es posible distinguir en función al modelo de negocio concreto, esto es B2C (de empresa a consumidor, extraído de los datos referidos a la variable "desplazarse en coche con conductor por la ciudad") o C2C (de consumidor a consumidor, con datos referidos a la variable "desplazarse en coche a otras ciudades aprovechando el viaje de un conductor particular"). No ocurre lo mismo en cuanto a los proveedores del servicio, de quienes no disponemos de datos en el modelo $\mathrm{B} 2 \mathrm{C}$ pero, dado el carácter profesional de dicha relación, carecería de relevancia en tanto se trata de una motivación claramente económica, puramente mercantil, como lo que motivaría cualquier tipo de negocio.

El fenómeno $\mathrm{C} 2 \mathrm{C}$, representado por plataformas como Blablacar, se caracteriza por dos rasgos básicos que condicionan el régimen jurídico de esta figura: los conductores que operan en dicha plataforma, ofrecen plazas libres en sus vehículos para compartir los gastos de desplazamiento de un viaje que van a realizar, y las cantidades que reciben los conductores sirven para sufragar los gastos que supone el propio trayecto, en ningún caso para obtener un lucro y por ello el conductor deberá asumir también su parte de los gastos y lo ingresado no podrá superar los costes del viaje. Sería una manifestación pura de lo que inicialmente se concibió como Economía Colaborativa.

Analizando la motivación de los consumidores de transporte colaborativo, nos encontramos con el papel protagonista de la motivación económica, ya sea participando en el modelo B2C $(59,00 \%)$ o participando en el modelo C2C (60,70\%). Respecto a la motivación ambiental, los consumidores del modelo B2C no se encuentran especialmente motivados por esta causa, ya que solo el 6,20\% de los encuestados manifiestan haberse decantado por utilizar este servicio colaborativo impulsados por esta razón, resultando lógico que no sea un porcentaje especialmente alto dada la dinámica de este modelo de servicio que, a efectos contaminantes, a priori no difiere de un servicio taxi convencional o de la utilización de vehículo privado; aunque sí se presentan unos mayores niveles de motivación ambiental en el modelo C2C, cuya dinámica es puramente la de compartir, que se registra como opción por un $19,40 \%$ de los encuestados.

En cuanto a las motivaciones sociales, al igual que sucedía en el ámbito ambiental, el modelo B2C registra poco peso de éstas $(3,20 \%)$, pero el modelo C2C constata unos niveles significativos, habiendo sido destacadas por el $16 \%$ de los encuestados.

Refiriendo a los proveedores del servicio de transporte en el modelo $\mathrm{C} 2 \mathrm{C}$, la motivación principal en su actuación es la económica $(59,50 \%)$, sin que ello suponga que los datos relativos tanto a motivaciones sociales $(28,00 \%)$ como a motivaciones ambientales $(26,70 \%)$ no sean significativos, destacando de ello que la motivación social pesa más en los individuos a la hora de ofrecer servicios de transporte que de cara a consumirlos.

Para una mejor comprensión, hemos ilustrado gráficamente las respuestas a dicha encuesta, tanto por parte de consumidores como de proveedores, agrupándolas en las diferentes tipologías de motivación en 
función a las respuestas dadas. En la figura 1 pueden observarse las motivaciones de los consumidores y en la figura 2 se encontrarían las motivaciones de los proveedores.

Figura 1. Motivaciones de los consumidores de transporte colaborativo, 2019. Porcentaje de individuos.

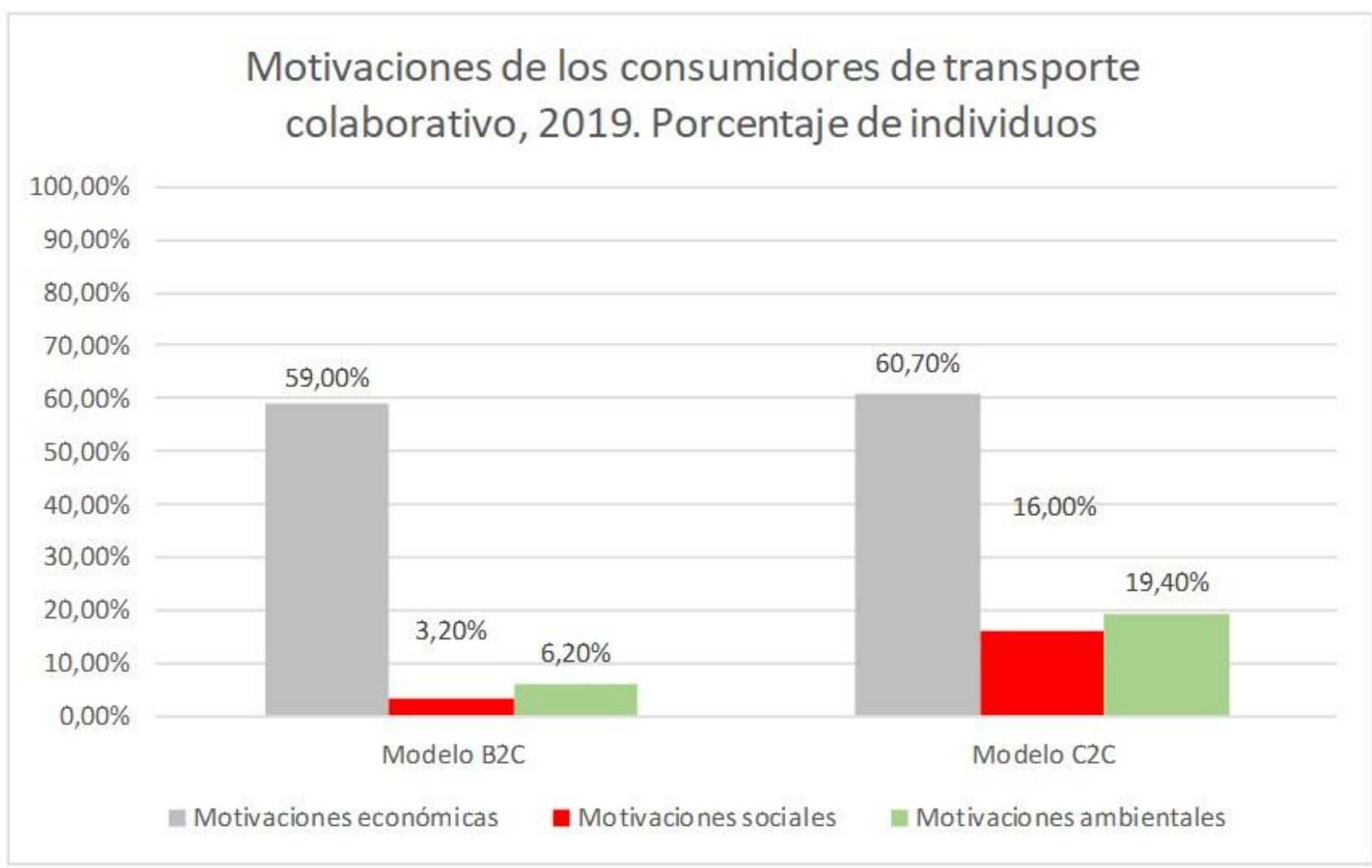

Fuente: Elaboración propia a partir de los datos de la encuesta "Panel de Hogares" elaborada por la CNMC para el año 2019.

Figura 2. Motivaciones de los proveedores de transporte colaborativo, 2019. Porcentaje de individuos.

\section{Motivaciones de los proveedores de transporte colaborativo, 2019. Porcentaje de individuos}

$100,00 \%$

$90,00 \%$

$80,00 \%$

$70,00 \%$

$60,00 \%$

$50,00 \%$

$40,00 \%$

$30,00 \%$

$20,00 \%$

$10,00 \%$

$0,00 \%$

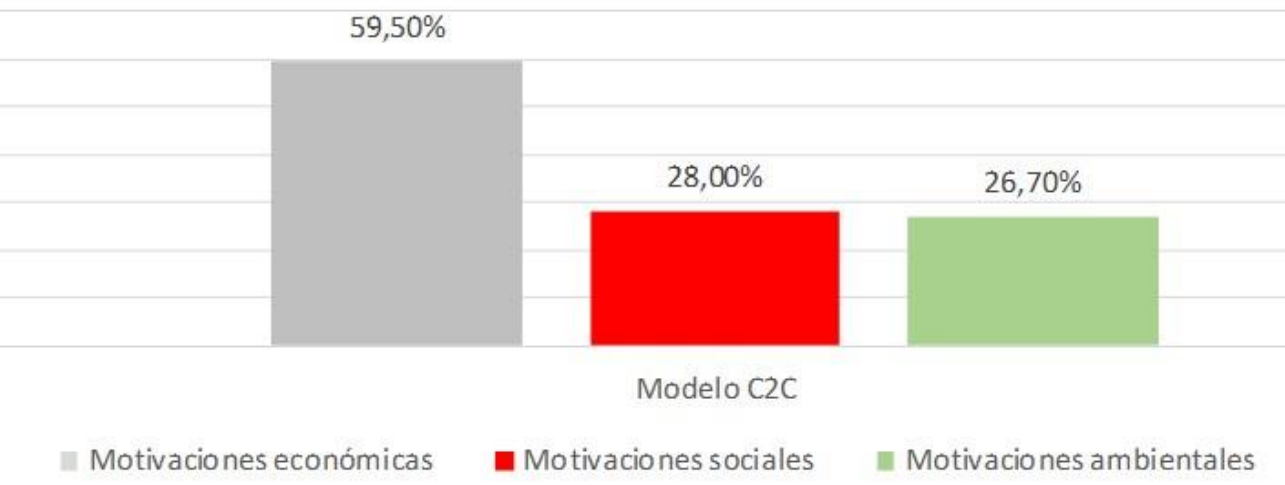

Fuente: Elaboración propia a partir de los datos de la encuesta "Panel de Hogares" elaborada por la CNMC para el año 2019. 
Por último, en cuanto al perfil del usuario español de transporte colaborativo, con datos de 2021, podemos afirmar que de forma mayoritaria la plataforma más consumida sería Uber (51\%), seguida por Cabify (48\%) y por el modelo C2C de Blablacar $(47 \%)^{6}$. Respecto al consumidor de Uber, demográficamente no se aprecia sesgo de género, mayoritariamente tendría entre 35 y 44 años (30\%) aunque el volumen de consumidores entre 25 y 34 años también es bastante reseñable (26\%) y preferentemente urbano (61\% frente al $39 \%$ rural y residente en pueblos) ${ }^{7}$. Por lo que respecta a Blablacar, la diferencia entre consumidores varones y mujeres es muy reducida (un 6\% más de hombres que de mujeres) ${ }^{8}$, los usuarios entre 25 y 34 años (33\%) son mayoritarios, seguido de los que tienen una edad comprendida entre 35-44 (27\%) , preferentemente urbanos $(55 \% \text { frente a } 45 \%)^{\mathbf{1 0}}$ y con un tamaño de hogar de 4 personas $(29 \%)^{\mathbf{1 1}}$.

\section{Conclusiones}

Doctrinalmente, resulta extremadamente complejo definir o delimitar los distintos modelos que van surgiendo y desarrollándose impulsados por las nuevas tecnologías al amparo de lo que se conoce como Economía Colaborativa. Aun así, habiendo optado por una definición institucional como es la conceptualización del Parlamento Europeo para enmarcar el objeto de este estudio, hemos comprobado que el factor social está indisolublemente ligado a este fenómeno, aunque su extensión sobrepase las dinámicas iniciales puras de cooperación y colaboración. De este modo, el ámbito social influye de forma crucial, tanto en la configuración de la decisión de participar como en los impactos derivados de ello; de la misma forma que, al centrar el estudio en el transporte colaborativo en España hemos determinado las específicas motivaciones y efectos de este servicio, sin generalizarlos para la EC en su conjunto.

Hemos complementado la literatura académica existente relativa a motivaciones y efectos para la participación (desde el lado de la oferta y la demanda) en los servicios colaborativos desde la óptica de la sostenibilidad (económicos, sociales y medioambientales) y del uso eficiente de los recursos disponibles.

Se ha demostrado empíricamente que, en el conjunto del país, la importancia de las motivaciones, cuando se actúan desde el lado de la demanda o desde el de la oferta, es distinta. Hemos analizado que los artículos académicos relativos a las motivaciones señalan que éstas no son excluyentes entre ellas y que varían en función del servicio concreto y de la posición del sujeto (oferente o demandante), lo que lleva a una aparente evidencia empírica contradictoria (Möhlmann, 2015; Luri et al., 2021). Tal es así que, incluso dentro de un mismo sector, el peso es distinto en las motivaciones cuando se actúan desde el lado de la demanda o desde el de la oferta, como hemos podido comprobar con el transporte colaborativo, así, en relación a los proveedores los motivos medioambientales sí son significativos, sin llegar al ser el motivo principal $(26,70 \%)$, registrando porcentajes superiores a los que se observaban para el caso de los consumidores.

Igualmente, este estudio ha demostrado que el modelo de uso del transporte colaborativo condicional claramente la motivación, mientras que en el modelo C2C (de consumidor a consumidor) son mucho más relevantes que en modelo B2C (de empresa a consumidor).

Por último, aportamos un perfil actual del usuario español de transporte colaborativo en cada una de las plataformas más consumidas en cada uno de estos dos modelos. En concreto, hemos apreciado que el género no es un rasgo distintivo en el uso de Uber, mientras que en de Blablacar el uso es mayor por parte de los hombres; el principal rango de edad de los usuarios de Uber es 35 y 44 años, frente a 25 y 34 años de Blablacar, mientras que los usuarios de ambas plataformas residen en zonas urbanas, si bien en Uber el porcentaje es considerablemente superior.

No existe pues una conclusión unánime dentro de la literatura académica sobre la valoración positiva o negativa de los efectos derivados del desarrollo de los diferentes negocios de economía colaborativa. Por ello, a través de la Teoría del Triple Balance hemos tenido ocasión de concluir que actualmente no es posible valorar como sostenible o no sostenible socialmente el fenómeno de la economía colaborativa. De la comparación y ponderación de los efectos descritos se ha podido extraer una situación de impasse, donde encontramos tanto efectos positivos dignos de mención como efectos negativos preocupantes. La decantación hacia un lado u otro derivará del propio trazado del modelo colaborativo, bien hacia formas más

6 Datos extraídos de Statista Global Consumer Survey, mayo de 2021, respuesta múltiple; Base: n=584 ride sharing users, respecto a la pregunta “¿Cuál de estos proveedores de transporte compartido has utilizado en los últimos 12 meses?”.

Datos extraídos de Statista Global Consumer Survey, mayo de 2021, respuesta única y respuesta múltiple; Base: n=296 Uber users, respecto a las preguntas “¿Cuál es su género?” y ¿En qué tipo de comunidad vive?”.

8 Datos extraídos de Statista Global Consumer Survey, mayo de 2021, respuesta única; Base: n=277 BlaBlaCar users, respecto a la pregunta “Cuál es su género?”.

9 Datos extraídos de Statista Global Consumer Survey, mayo de 2021, respuesta única; Base: n=277 BlaBlaCar users, respecto a la pregunta “¿Qué edad tiene?"

10 Datos extraídos de Statista Global Consumer Survey, mayo de 2021, respuesta única; Base: n=277 BlaBlaCar users, respecto a la pregunta “ ¿En qué tipo de comunidad vive?"

11 Datos extraídos de Statista Global Consumer Survey, mayo de 2021, respuesta única; Base: n=277 BlaBlaCar users, respecto a la pregunta “Cuánta gente, incluido usted mismo y todos los niños, viven permanentemente en su hogar?”. 
profesionalizadas (como Uber) o bien hacia un retorno a la compartición inicial entre iguales (como Blablacar). Se ha constatado que las plataformas digitales, como herramienta, son capaces de maximizar beneficios a costa del entorno local pero también se pone la innovación al servicio de las personas y la sociedad, dependiendo de los valores y principios que la fundamenten (Dieste, 2020).

El resultado de este análisis proporciona un marco de trabajo a investigaciones teóricas futuras, en tanto construye matrices sociales que contraponen los aspectos positivos frente a los efectos negativos de este paradigma; al tiempo que podría consistir una herramienta práctica en tanto perfila al usuario de transporte colaborativo en España, su caracterización y qué le mueve a participar, lo que facilitaría el lanzamiento de campañas destinadas a los grupos sociodemográficos de interés en cuestión.

Todo lo cual, hace oportuno continuar con los estudios sobre efectos y motivación en EC desde la perspectiva de la sostenibilidad, con el fin de conocer mejor las razones que llevan a consumidores y proveedores a utilizar estos modelos de negocio y cuál es el peso real de las motivaciones y las consecuencias de sus efectos en función de los distintos servicios colaborativos. El impulso de nuevos análisis nos permitirá hacer un uso más eficiente de los recursos disponibles a través de las cada vez más significativas plataformas colaborativas.

\section{Referencias bibliográficas}

Abhari, K., Davidson, E. K. y Xiao, B. (2019) Collaborative innovation in the sharing economy Profiling social product development actors through classification modelling. Internet Research, 29(5), pp. 1014-1039. DOI 10.1108/INTR03-2018-0129.

Ahuja, R. y Lyons, R. (2017) The Silent Treatment: LGBT Discrimination in the Sharing Economy. Trinity Economics Papers tep 1917, Trinity College Dublin, Department of Economics.

Albinsson, P. A. y Perera, B. Y. (2012) Alternative marketplaces in the 21st century: Building community through sharing events. Journal of consumer Behaviour, 11(4), pp. 303-315.

Aparici Marino, R. y García Marín, D. (2018) Prosumidores y emirecs: Análisis de dos teorías enfrentadas. Comunicar: Revista científica iberoamericana de comunicación y educación, 55, pp. 71- 79. DOI: 10.3916/C55-2018-07.

Ballester Casanella, B. (2019) La protección de los trabajadores en el marco de la Economía Colaborativa y en las plataformas digitales. R.E.D.S., 15.

Barnes, S. J. y Mattson, J. (2016) Understanding Current and Future Issues in Collaborative Consumption: A FourStage Delphi Study. Technological Forecasting and Social Change, 104. DOI:10.1016/j.techfore.2016.01.006.

Barnes, S. J. y Mattsson, J. (2017) Understanding Collaborative Consumption: Test of a Theoretical Model. Technological Forecasting and Social Change, 118@, pp. 281-292. DOI: 10.1016/j.techfore.2017.02.029.

Benkler, Y. (2004) Sharing Nicely: On Shareable Goods and the Emergence of Sharing as a Modality of Economic Production. The Yale Law Journal, 114, pp. 273-358.

Bicchieri, C., Duffy, G. y Tolle, G. (2004) Trust Among Strangers. Philosophy of Science, 71, pp. 1-34.

Binninger, A. S., Ourahmoune, N. y Robert, I. (2015) Collaborative Consumption and Sustainability: A Discursive Analysis of Consumer Representations and Collaborative Website Narratives. Journal of Applied Business Research (JABR), 31(3), pp. 969-986 .

Birke, D. (2009) The economics of networks: a survey of the empirical literatura. Journal of Economic surveys, 23(4).

Böcker, L. y Meelen, T. (2017) Sharing for people, planet or profit? Analysing motivations for intended sharing economy participation. Environmental Innovation and Societal Transitions 23, pp. 28-39. https://doi.org/10.1016/j.eist.2016.09.004.

Botsman, R. y Rogers, R. (2010) Whats mine is yours: The rise of collaborative consumption. Glasgow, UK: Collins Bussiness.

Cagle, S. (2014) The case against sharing. (acceso 24.08.2021) https://medium.com/the-nib/the-case-against-sharing9ea5ba3d216d.

Canniford, R. (2011) A Tipology of Consumption Communities. Research in Consumer Behavior, 13, pp. 57-75.

Cansoy, M. y Schor, J. (2016). Who Gets to Share in the «Sharing Economy»: Understanding the Patterns of Participation and Exchange in Airbnb". Unpublished Paper, Boston College.

Cherry, C. E. y Pidgeon, N. F. (2018) Is Sharing the Solution? Exploring Public Acceptability of the Sharing Economy. Journal of Cleaner Production, 195, 939-948. Doi:10.1016/j.jclepro.2018.05.278.

CNMC (2016) Conclusiones preliminares sobre los nuevos modelos de prestación de servicios y la economía colaborativa. http://ka-au.net/wp-content/uploads/2017/01/05.CNMC_GOVERNMENT_PRO_SP.pdf.

CNMC (2019) Paneldehogares. http://data.cnmc.es/datagraph/ (acceso 26.08.2021).

Comisión Europea (2016) Una agenda europea para la Economía Colaborativa. COM(2016) 356 final. https://eurlex.europa.eu/legal-content/ES/TXT/PDF/?uri=CELEX:52016DC0356\&from=ES.

Cova, B. y Cova, V. (2002) Tribal marketing: The tribalisation of society and its impact on the conduct of marketing. European Journal of Marketing, 36(5/6): 595-620. DOI:10.1108/03090560210423023.

Cova, B. y White, T. (2010) Counter-brand and alter-brand communities: The impact of Web 2.0 on tribal marketing approaches. Journal of Marketing Management, 26(3): 256-270. DOI:10.1080/02672570903566276. 
Cui, R., Li, J. y Zhang, D.J. (2016) Discrimination with incomplete information in the sharing economy: Field evidence from Airbnb. Management Science. DOI: 10.2139/ssrn.2882982 .

Díaz- Foncea, M., Marcuello Servós, C., y Montreal Garrido, M. (2016) Economía social y Economía Colaborativa: Encaje y potencialidades. Economía Industrial, 402, pp 27- 35.

Dieste Cobo, J.M. (2020) Las plataformas colaborativas como oportunidad para la innovación social. REVESCO. Revista de Estudios Cooperativos, vol. 133, e67338. https://dx.doi.org/10.5209/REVE.67338.

Dip, J.A; Simes, H.; Benítez, J.P. (2020) Las Cooperación y reciprocidad en la economía colaborativa de Airbnb. Un estudio para la provincia turística de Misiones, Argentina. REVESCO. Revista de Estudios Cooperativos, vol. 135, e69176. https://dx.doi.org/10.5209/reve.69176.

Dredge, D. y Gymóthy, S. (2015) The Collaborative Economy and Tourism: Critical Perspectives, Questionable Claims and Silenced Voices. Tourism Recreation Research, 40(3), pp. 286-302. DOI:10.1080/02508281.2015.1086076.

Edelman, B., Luca, M. y Svirsky, D. (2017) Racial Discrimination in the Sharing Economy: Evidence from a Field Experiment. American Economic Journal: Applied Economics, 9(2), pp. 1-22.

Elkington, J. (2001) The triple bottom line for 21 st century business. Welford, R. y Starkey, R. (ed.). The Earthscan reader in business and sustainable development. London: Taylor \& Francis. pp. 23-43.

EY-Parthenon (2020) Deconstruyendo al consumidor. https://assets.ey.com/content/dam/ey-sites/eycom/es_es/topics/consumer-products/ey-informe-completo-deconstruyendo-al-consumidor.pdf.

Ferrari, M. (2016) Beyond Uncertainties in the Sharing Economy: Opportunities for Social Capital. European Journal of Risk Regulation, 7(4), pp. 664-674. DOI:10.1017/S1867299X00010102.

Ferrell, O. C. y Hirt, G. (2003) Introducción a los negocios en un mundo cambiante. Nueva York: McGraw-Hill.

Ferrer, M y Paniagua, E. (2017) Trabajo en plataformas digitales, Análisis y propuestas de regulación, GoVup/Adigital. http://grupo.us.es/iwpr/wp-content/uploads/2016/09/GOVUP-Trabajo-en-plataformas-digitales.pdf.

Fischer, S. L., Pahus, H. S., y Bager, A. (2019) What motivates people to become Airbnb hosts - do we know enough? - an exploration of the literature. Research in Hospitality Management, 9:2, pp. 83-88. DOI: $10.1080 / 22243534.2019 .1689697$.

Foucault, M. (2007) Nacimiento de la biopolítica. Buenos Aires: Fondo de Cultura Económica.

Frenken, K. (2017) Political Economies and Environmental Futures for the Sharing Economy. Philosophical Transactions of the Royal Society, 375.

Frenken, K., y Schor, J. (2017) Putting the sharing economy into perspective. Environmental Innovation and Societal Transitions, 23.

Germann Molz, J. (2013) Social Networking Technologies and the Moral Economy of Alternative Tourism: The Case of Couchsurfing.Org. Annals of Tourism Research, 43, pp. 210-230. DOI: 10.1016/j.annals.2013.08.001.

Gil, J. (2016) Economías colaborativas y crisis del capitalismo: un análisis a través de la prosumición. En Cotarelo, R. y Gil, J. (Eds.). Ciberpolítica: gobierno abierto, redes, deliberación, democracia. 167-188. Madrid: Instituto Nacional de Administración Pública.

Gil, J. y Sequera, J. (2018) Expansión de la ciudad turística y nuevas resistencias. El caso de Airbnb en Madrid. EMPIRIA. Revista de Metodología de Ciencias Sociales, 41, pp. 15-32. DOI/empiria.41.2018.22602.

Gold, L. (2010) EC New financial horizons: the emergence of an Economy of Communion. New York: New York City Press.

Goldin, A. (2019) El Derecho del Trabajo, hoy. Tendencias y desafíos. En Caparrós, L. y García H. O. (coord). El trabajo en la economía de plataformas. Buenos Aires: Ediar.

González, S., y Waley, P. (2013) Traditional retail markets: The new gentrification frontier? Antipode, 45(4). DOI:10.1111/j.1467-8330.2012.01040.x.

González de Rivera Outomuro, J. (2019) Sociología de las instituciones digitales: El estudio de la Economía Colaborativa. Tesis doctoral. Madrid: Universidad Complutense de Madrid.

Gottlieb, C. (2013) Residential Short-Term Rentals: Should Local Governments Regulate the'Industry'?. Planning and Environmental Law, 65(2), pp. 4-9.

Gualtieri T. (2014): la economía colaborativa se afianza. El País. https://bit.ly/2X22LbK.

Guttentag, D., Smith, S., Potwarka, L. y Havitz, M. (2018) Why tourists choose Airbnb: A motivation-based segmentation study. Journal of Travel Research, 57(3), pp. 342-359.

Hajibaba, H. y Dolnicar, S. (2017) Do Hosts Discriminate?. En Dolnicar, S. (coord). Peer-to-Peer Accommodation Networks: Pushing the boundaries. Oxford: Goodfellow Publishers, pp. 215- 224.

Hamari, J., Sjöklint, M. y Ukkonen, A. (2016) The sharing economy: Why people participate in collaborative consumption. Journal of the association for information science and technology, 67(9), pp. 2047-2059.

Hawlitschek, F.; Teubner, T.; y Gimpel, H. (2018) Consumer motives for peer-to-peer sharing. Journal of Cleaner Production, 204, pp. 144-157.

Hindle, T. (2009) Triple bottom line. The Economist. (acceso el 28.08.2021) https://www.economist.com/news/2009/11/17/triple-bottom-line.

Janoschka, M. y Sequera, J. (2016). Gentrification in Latin America: addressing the politics and geographies of displacement. Urban Geography, 37(8), pp. 1-20. DOI:10.1080/02723638.2015.1103995.

Kankanhalli, A., Tan, B. C. y Wei, K. K. (2005) Contributing knowledge to electronic knowledge repositories: An empirical investigation. MIS quarterly, 29(1). 
Kassan, J. y Orsi, J. (2012) The Legal Landscape of the Sharing Economy. Journal of Environmental Law and Litigation, 27(1).

Kilhoffer, Z., Lenaerts, K. y Beblabý, M. (2017) The Platform Economy and Industrial Relations Applying the old framework to the new reality. CEPS Research 12. https://papers.ssrn.com/sol3/papers.cfm?abstract id=3053826.

Kim, E. y Yoon, S. (2021) Social capital, user motivation, and collaborative consumption of online platform services. Journal of Retailing and Consumer Services, 6. https://doi.org/10.1016/j.jretconser.2021.102651.

Labrecque, L. I., Esch, J. V. D., Mathwick, C., Novak, T. P. y Hofacker, C. F. (2013) Consumer Power: Evolution in the Digital Age. Journal of Interactive Marketing, 27(4), pp. 257-269.

Lacalle, D. (2009) Trabajadores precarios, trabajadores sin derechos, Madrid: Editorial El viejo topo.

Lamberton, C. P. y Rose, R. L. (2012) When is ours better than mine? A framework for understanding and altering participation in commercial sharing systems. Journal of Marketing. 76(4), pp. 109-125.

Luffiego García, M. (2000) La evolución del concepto de sostenibilidad y su introducción en la enseñanza. Historia y Epistemología de las Ciencias, 18(3), pp. 473-486.

Luri, A., Ramos, C., y Bruscato, A. (2021) Sharing economy versus collaborative consumption: What drives consumers in the new forms of exchange?. Journal of Business Research, 128, pp. $124-137$. https://doi.org/10.1016/j.jbusres.2021.01.035.

Martin, C. J. (2016) The Sharing Economy: A Pathway to Sustainability or a Nightmarish Form of Neoliberal Capitalism?. Ecological Economics, 121, pp. 149-159. DOI:10.1016/j.ecolecon.2015.11.027.

Martin, C. J. y Upham, P. (2016) Grassroots social innovation and the mobilisation of values in collaborative consumption: a conceptual model. Journal of Cleaner Production, 134(A). pp. $204-213$. https://doi.org/10.1016/j.jclepro.2015.04.062.

McKay, S., Jefferys, S et al. (2012) Study on Precarious work and social rights. London: Working Lives Research Institute.

Menor- Campos, A., García- Moreno, M. B., López- Guzmán, T. y Hidalgo- Fernández, A. (2019) Effects of Collaborative Economy: A Reflection. Social Sciences, 8, 142, DOI: 10.3390/socsci8050142.

Mertens Horn, K. (2017) Is Home Sharing Driving Up Rents? Evidence from Airbnb in Boston. Journal of Housing Economics, 38. DOI:10.1016/j.jhe.2017.08.002.

Möhlmann, M. (2015) Collaborative consumption: determinants of satisfaction and the likelihood of using a sharing economy option again. Journal of Consumer Behaviour, 14(3), pp. 193-207.

Morozov, E. (2013) The ,sharing economy' undermines workers rights. Financial Times, Opinion Ecommerce. (acceso 05.09.2021), disponible en: https://evgenymorozov.tumblr.com/post/64038831400/the-sharing-economyunderminesworkers-rights.

Munar, A. M. y Gyimóthy, S. (2013) Critical Digital Tourism Studies. Tourism Social Science Series, 18, pp. $245-311$. DOI: $10.1108 / \mathrm{S} 1571-5043(2013) 0000018016$.

Muniz, A. M. y O’Guinn, T. C. (2001) Brand Community. Journal of Consumer Research, 27, pp. 412-432. https://doi.org/10.1086/319618.

Närvänen, E., Kartastenpää, E. y Kuusela, H. (2013) Online lifestyle consumption community dynamics: A practice-based analysis. Journal of Consumer Behaviour, 12(5), pp. 358-369.

Nevo, A., Turner, J., y Williams, J. (2016) Usage-based pricing and demand for residential broadband. Econometrica, 84(2), pp. 411-443.

Nofre, J., Giordano, E., Eldridge, A., Martins, J. C., \& Sequera, J. (2018) Tourism, nightlife and planning: challenges and opportunities for community liveability in La Barceloneta. Tourism Geographies, 20(3), pp. 377-396. DOI: 10.1080/14616688.2017.1375972.

Owyang, J., Tran, C., y Silva, C. (2013) The collaborative economy. United States: Altimeter http://www.collaboriamo.org/media/2014/04/collabecon-draft16-130531132802-phpapp02-2.pdf.

Ostrom, E. (2010) Beyond Markets and States: Polycentric Governance of Complex Economic Systems. American Economic Review, № 100, pp. 641-672.

Parlamento Europeo (2017a). Informe sobre una Agenda Europea para la Economía Colaborativa (2016/0000(INI). https://www.europarl.europa.eu/doceo/document/A-8-2017-0195 ES.html.

Parlamento Europeo (2017b) Resolución, de 19 de enero de 2017, sobre un pilar europeo de derechos sociales (2016/2095(INI). https://www.europarl.europa.eu/doceo/document/TA-8-2017-0010_ES.html.

Patiño, D.; Gómez-Álvarez R. y Plaza, J. J. (2017) Caracterización económica y cuantificación de la Economía Colaborativa. Rodríguez-Piñero, M. (dir.) y Hernández-Bejarano, M. (dir.). Economía colaborativa y trabajo en plataforma: realidades y desafíos. Albacete (España): Editorial Bomarzo, 51-101.

Peitz, M. y Waldfogel, J., (eds.) (2012) The Oxford Handbook of the Digital Economy. Oxford: Oxford University Press.

Pereira, T. H. M., y Martins, H. C. (2021) People, Planet, and Profit: A Bibliometric Analysis of Triple Bottom Line Theory. Journal of Management and Sustainability, 11(1), pp. 1-64.

Peters, C., Bodkin, C. D., y Fitzgerald, S. (2012) Toward an understanding of meaning creation via the collective co-production process. Journal of Consumer Behaviour, 11(2), pp. 124-135.

Peterson, N. A., Speer, P. W. y McMillan, D. W. (2008) Validation of a brief sense of community scale: Confirmation of the principal theory of sense of community. Journal of community psychology, 36(1), pp. 61-73. 
Pick, F. (2012) Building Trust in Peer-to-Peer Marketplaces: An Empirical Analysis of Trust Systems for the Sharing Economy. AV Akademikerverlag.

Pixová, M. y Sládek, J. (2016) Touristification and awakening civil society in postsocialist Prague. En Colomb, C. y Novy, J. (ed.) Protest and resistance in the tourist city. London: Routledge, 73-89.

Prettenthaler, F. E. y Steininger, K. (1999) From ownership to service use lifestyle: the potential of car sharing. Ecological economics, 28(3), pp. 443-453.

Rifkin, J. (2014) The Zero Marginal Cost Society: The Internet of Things, the Collaborative Commons, and the Eclipse of Capitalism, London: Palgrave Macmillan.

Ríos- Osorio, L., Cruz- Barreiro, I. y Welsh- Rodríguez, C. (2013) The Concept of Sustainable Development from an Ecosystem Perspective: History, Evolution, and Epistemology, en Yañez-Arancibia, A., Dávalos-Sotelo, R., Day, J., Reyes, E., Ecological Dimensions for Sustainable Socio Economic Development. Southampton, Boston: Wit Press, pp. 29-46.

Robbins, S. P. y Coulter, M. (2005) Administración. Nueva Jersey: Pearson Prentice Hall.

Rogers, B. (2017) The social costs of Uber. University of Chicago Law Review online, 82(1) art 6.

Sánchez Fernández, G. (2009) Análisis De La Sostenibilidad Agraria Mediante Indicadores Sintéticos: Aplicación Empírica Para Sistemas Agrarios. Tesis Doctoral. Madrid: Universidad Politécnica de Madrid.

Scaraboto, D. (2015) Selling, Sharing, and Everything In Between: The Hybrid Economies of Collaborative Network. Journal of Consumer Research. 42(1), pp. 152-176. https://doi.org/10.1093/jcr/ucv004.

Scholz, T. (2014) Platform Cooperativism vs. the Sharing Economy. (acceso el 25.08.2021) https://medium.com/@trebors/platform-cooperativism-vs-the-sharing-economy-2ea737f1b5ad.

Schor, J. (2017) Does the Sharing Economy Increase Inequality Within the Eighty Percent?: Findings from a Qualitative Study of Platform Providers. Cambridge Journal of Regions, Economy and Society, 10(2).

Schor, J. y Attwood-Charles, W. (2017) The "sharing" economy: labor, inequality, and social connection on for-profit platforms. Sociology Compass, 11(8).

Schor, J. y Fitzmaurice, C. (2015) Handbook on Research on Sustainable Conumption. Cheltenham: Edward Elgar.

Sequera, J. (2017) Ante una nueva civilidad urbana. Capitalismo cognitivo, habitus y gentrificación. Revista Internacional de Sociología, 75(1).

Smith, A. (2016) Shared, Collaborative and on Demand: The New Digital Economy. (acceso 27.08.2021) http://www.pewinternet.org/2016/05/19/the-new-digital-economy/.

Soler Porro, A.B. y Chirolde Núñez, R.R. (2010) Motivación y rendimiento docentes en estudiantes bolivianos del Nuevo Programa de Formación de Médicos. Educación Médica Superior, 24 (1), pp. 42-51.

Sundararajan, A. (2016) The Sharing Economy. The End of Employment and the Rise of Crowd-based Capitalism. Cambridge: MIT Press.

Sutherland, W. y Jarrahi, M. H. (2018) The Sharing Economy and Digital Platforms: A Review and Research Agenda. International Journal of Information Management, 43, pp. 328-341. DOI:10.1016/j.ijinfomgt.20ol18.07.004.

Toni, M., Renzi, M. F. y Mattia, G. (2018) Understanding the Link Between Collaborative Economy and Sustainable Behaviour: An Empirical Investigation. Journal of Cleaner Production, 172, $4467-4477$. DOI:10.1016/j.jclepro.2017.11.110.

Tussyadiah, I. P. (2016) Factors of Satisfaction and Intention to Use Peer-to-Peer Accommodation. International Journal Hosp. Manag, 55, pp. 70-80.

Tussyadiah, I. P. y Pesonen, J. (2018) Drivers and Barriers of Peer-to-Peer Accommodation Stay- An Exploratory Study with American and Finnish Travellers. Current. Issues Tour, 21, pp. 703-720 .

Wachsmuth, D. y Weisler, A. (2018) Airbnb and the rent gap: Gentrification through the sharing economy. Environment and Planning A: Economy and Space, 50(6), pp. 1147-1170. https://doi.org/10.1177/0308518X18778038.

Wosskow, D. (2014) Unlocking the sharing economy. An independent review. Department of businesses, innovation and skills. London: UK Government.

Zelizer, V. A. (2010) Economic Lives: How Culture Shapes the Economy. Princeton, New Jersey: Princeton University Press. 